\title{
Prevalence of Cerebral Atherosclerosis among Patients with Metabolic Syndrome: A Case Control Study on Egyptian Subjects
}

\author{
Sherif Elwan', Tarek Zoheir Tawfik², Heba Sayed Assal1, Shaimaa El-Jaafary², \\ Montasser Mohamed Hegazy², Salwa Tawfik1, Alshaimaa Mahmoud Aboulfotooh2, \\ Foad Abd-Allah ${ }^{*}$ \\ ${ }^{1}$ Department of Internal Medicine, National Research Institute, Cairo, Egypt \\ ${ }^{2}$ Department of Neurology, Cairo University, Cairo, Egypt \\ Email: "foad.abdallah@kasralainy.edu.eg
}

Received 4 December 2015; accepted 24 January 2016; published 27 January 2016

Copyright (C) 2016 by authors and Scientific Research Publishing Inc.

This work is licensed under the Creative Commons Attribution International License (CC BY). http://creativecommons.org/licenses/by/4.0/

(c) (i) Open Access

\begin{abstract}
Background: The metabolic syndrome (MetS) is a clustering of vascular risk factors that tend to increase the risk of occurrence of diabetes mellitus type 2 (DMT2), cardiovascular and cerebrovascular ischemic events. The aim of the present study is to investigate the association of extracranial and intracranial arterial atherosclerosis as well as silent brain infarction (SBI) with MetS, thereby determining the potential cerebrovascular atherosclerotic risk of MetS in the Egyptian population. Methods: A case control study was conducted on 50 Egyptian subjects with MetS and 30 without with age range from 40 - 60 years old. All participants were free from cerebrovascular ischemic events [stroke or transient ischemic attack (TIA)]. All participants underwent complete neurological examination, assessment of the diagnostic criteria for MetS, carotid and transcranial duplex ultrasonography (U/S) and brain MRI. Results: Preclinical carotid atherosclerosis (atherosclerotic plaques with $<\mathbf{5 0 \%}$ stenosis) was associated with MetS (P value $=\mathbf{0 . 0 2}$ ) that persisted after adjustment for age and other confounders. There was no significant association between increased intima media thickness (IMT) and MetS. There was non-significant association between MetS and intracranial atherosclerotic disease (ICAD) or the degree of intracranial stenosis (ICS). Conversely, there was a highly significant association between MetS and SBI even after adjustment for age and other confounders $(P$ value $=\mathbf{0 . 0 0 1}$ ). Conclusion: Metabolic syndrome is an important factor associated with mild to moderate atherosclerosis $(<50 \%$ stenosis $)$ and silent brain infarcts among asymptomatic individuals. Interventions to reduce MetS are important for prevention of subclinical and clinical cerebral atherosclerotic disease.
\end{abstract}

${ }^{*}$ Corresponding author.

How to cite this paper: Elwan, S., Tawfik, T.Z., Assal, H.S., El-Jaafary, S., Hegazy, M.M., Tawfik, S., Aboulfotooh, A.M. and Abd-Allah, F. (2016) Prevalence of Cerebral Atherosclerosis among Patients with Metabolic Syndrome: A Case Control Study on Egyptian Subjects. World Journal of Cardiovascular Diseases, 6, 8-13. http://dx.doi.org/10.4236/wjcd.2016.61002 


\section{Keywords}

Metabolic Syndrome, Preclinical Atherosclerosis, Silent Brain Infarction, Neurovascular Ultrasound, Brain MRI, Egyptian Population

\section{Introduction}

Metabolic Syndrome (MetS) is a cluster of interconnected factors that directly increase the risk of vascular atherosclerosis and diabetes mellitus type 2 (DMT2) [1].

The most recent definition of metabolic syndrome is the joint interim statement (consensus definition) adopted by the International Diabetes Federation (IDF), American Heart Association (AHA) and the National Heart, Lung and Blood institute (NHLBI) [2].

The main components of MetS are raised blood pressure, a high fasting blood glucose level, abdominal obesity and increase triglycerides levels and low HDL cholesterol that occurs together [3].

It is not known whether this entity represents a specific syndrome or is a surrogate of combined risk factors that put the individual at particular risk [1]).

Metabolic syndrome accelerates the course of atherosclerosis. Detecting carotid artery as early as possible by vascular ultrasound is helpful to primary prevention of cardiovascular events in the patients with metabolic syndrome.

The risk of MetS on cerebral large and small artery atherosclerosis in asymptomatic subjects has been assessed by a number of investigators. While many of them concluded that MetS had a significant risk on carotid atherosclerosis and silent brain infarct (SBI), some authors found out that the syndrome did not constitute such a separate risky entity [4]-[8]. The association of metabolic syndrome with stroke is extremely important. More than $22 \%$ of American men and 24\% of American women over age 20 are estimated to have metabolic syndrome. Recognition and treatment of this syndrome may lead to decreased stroke risk, improved cognitive function, and better quality of life as the population continues to age.

The aim of this study is to address the potential cerebrovascular atherosclerotic risk of MetS among sample of the Egyptian population.

\section{Materials and Methods}

Study design: A case control study.

\subsection{Study Population}

The study was conducted on 80 subjects aged 40 - 60 years old with variable vascular risk factors (DM, HTN, dyslipidemia and/or abdominal obesity) from the internal medicine \& diabetes mellitus outpatient clinics of Kasr El Ainy hospital, Cairo University, All subjects were asymptomatic regarding cerebrovascular events. We further subdivided our group into 50 subjects fulfilling MetS diagnostic criteria (having 3 or more of the 5 diagnostic criteria) and 30 subjects not fulfilling them (having 1 or 2 of the diagnostic criteria) [2].

We excluded patients with current or previous history of cerebrovascular events (stroke or TIAs), patients with non-atherosclerotic Carotid disease (systemic vasculitis, radiation arteritis etc.), cardiac patients (such as atrial fibrillation, ischemic heart disease or cardiomyopathy) and patients with difficult insonation due to poor transtemporal bone window.

The study was approved by the institutional ethical committee, and all patients provided informed consent.

All subjects of the study underwent the following:

\section{Assessment of vascular risk factors for diagnosis of MetS}

Through laboratory investigations (fasting plasma glucose and complete lipid profile: TG, total cholesterol, HDL-C \& LDL-C plasma levels), blood pressure measurement and assessment of obesity; carried out by measurement of waist circumference (which is measured by applying the tape measure around the waist midway between the lowest point of the lower costal rib and the iliac crest on both sides of the abdomen, WC is measured at the end of expiration). According to the consensus definition of the MetS and cut off set by theinterna- 
tional diabetes federation (IDF). Abdominal obesity was diagnosed when waist circumference was $\geq 94 \mathrm{~cm}$ in men $\& \geq 80 \mathrm{~cm}$ in women.

\section{Imaging}

A. Vascular Imaging: All patients were subjected to Duplex Ultrasonography for extra and intra cranial vasculature at the Neurovascular Ultrasound Laboratory in the Neurology Department, Cairo University using Phillips HDI 5000 ultrasound equipment.

\section{1) Extracranial vessels}

Carotid duplex scanning was performed by qualified vascular operators using Philips HDI 5000 machines. A high frequency (7 - $10 \mathrm{MHz}$ ) linear array transducer was employed to scan the carotid from the most proximal CCA to the ICA as far as the mandible permitted. The identified parameters and measures named: Intima Media Thickness (IMT), presence of carotid plaque, degree of carotid stenosis and occlusion. The measures and quantifications of extra-cranial carotid atherosclerosis were performed according to the internationally published data.

\section{2) Transcranial Color Coded Doppler Ultrasonography (TCCS) technique}

Transcranial color-coded duplex ultrasonography was performed using a 2 - $4 \mathrm{MHz}$ phased-array transducer through the following bone windows:

A: Temporal window to assess both right and left middle cerebral arteries (MCAs), anterior cerebral arteries (ACAs), posterior cerebral arteries (PCAs) as well as the intracranial part of internal carotid arteries (ICAs).

B: Sub occipital window to assess the basilar artery (BA) and intracranial right and left vertebral arteries (VAs) through the foramen magnum.

Diagnosis of intracranial stenosis interpreted according to the internationally published criteria.

B. Brain Imaging: MRI of the brain parenchyma was performed on 45 subjects (24 diagnosed with MetS \& 21 not having the syndrome) using a 1.5 T Philips scanner at the radiodiagnosis department, Kasr El Ainy hospital, Cairo university. Sequences used were axial T1, T2, FLAIR \& DWI. According to DWI findings, we further classified these subjects into group 1 having SBIs \& group 2 not having SBIs. SBIs were diagnosed as foci $\geq 3 \mathrm{~mm}$. and $<1 \mathrm{~cm}$. in maximal diameter, of altered signal intensity (hyperintense in T2 and FLAIR and iso or hypointense in $\mathrm{T} 1$ compared to the white matter), located anywhere in the white matter (subcortical, capsular, periventricular, cerebellar or brain stem), not in the location of known prominent perivascular spaces and with no mass effect or perifocaloedema.

\subsection{Statistical Analysis}

All collected data were revised for completeness and consistency. Pre-coded data was entered on the computer using "Microsoft Office Excel Software” program (2013) for windows. Data was then transferred to the Statistical Package of Social Science Software program, version 21 (SPSS) to be statistically analysed. Data was summarized using mean, and standard deviation for quantitative variables and frequency and percentage for qualitative ones. Comparison between groups was performed using independent sample t-test for quantitative variables and Chi square or Fisher's exact test for qualitative ones. Standard logistic regression models were conducted to explore the significant determinants of abnormal MRI findings \& plaques among MetS patients, as well as to determine which investigation would be the most specific to predict MetS. P values $\ll 0.05$ were considered statistically significant, and $\ll 0.01$ were considered highly significant. Graphs were used to illustrate some information.

\section{Results}

We analyzed the data of 80 subjects with vascular risk factors (DM, HTN, dyslipidemia and/or abdominal obesity), none of which showed history or symptoms of cerebrovascular stroke or TIAs. Their age ranged from 40 60 years old with a mean age of $50.7 \pm$ standard deviation (SD) 7.1 years. They were divided into group 1 [50 subjects (62.5\%) fulfilling diagnostic criteria for MetS] and group 2 [30 subjects (37.5\%) having only 1 or 2 vascular risk factors (thus not diagnosed with MetS)].

Extracranial carotid duplex scanning showed plaques with low grade stenosis $(<50 \%$ stenosis) in $19(38 \%)$ patients in group 1 and $4(13.3 \%)$ patients in group $2(\mathrm{P}$ value $=0.02)$ while mean IMT was $0.09 \mathrm{~cm}$. in group 1 and $0.08 \mathrm{~cm}$. in group 2 ( $\mathrm{P}$ value $=0.053$ ). In group 1 plaques were unilateral in $12(63.2 \%)$ patients and bilateral in 7 (36.8\%) patients, while in group 2 all $4(100 \%)$ patients had unilateral plaques ( $\mathrm{P}$ value $=0.3)$. None of the subjects included in the study showed high grade stenosis ( $>50 \%$ stenosis). In group 1 , we found 1 patient 
having Lt. V2 segment occlusion with retrograde flow from the ipsilateral V4 segment.

Transcranial colour coded duplex scanning revealed intracranial atherosclerotic disease ICAD to exist in 8 (16\%) patients in group 1 and $3(10 \%)$ patients in group 2 (P value $=0.5)$. In group 1, 7 (87.5\%) patients had $<50 \%$ intracranial stenosis and $1(12.5 \%)$ patient had $>50 \%$ stenosis, while in group 2 all $3(100 \%)$ patients had $<50 \%$ stenosis ( $\mathrm{P}$ value $=1$ ). Regarding the number of diseased vessels, it showed variable distribution among the participants, group 1 showed; 4 (50\%) patients had single vessel disease and 4 (50\%) patients had multiple vessel disease, on the other hand group 2 revealed 2 (66.7\%) patients with single vessel disease and $1(33.3 \%)$ patient with multiple vessel disease $(\mathrm{P}$ value $=1)$.

Out of the 80 subjects enrolled in the study, we were able to perform brain MRI for 45 subjects (24 in group 1 and 21 in group 2). In group 1, 18 (75\%) patients had SBI and 6 (25\%) patients had normal MRI scan. Group 2 showed 5 (23.8\%) patients having SBI and 16 (76.2\%) patients with normal MRI scan (P value $=0.001)$. Regarding the number of lesions found on MRI, group 1 had 7 (38.9\%) patients with a single lesion and 11 (61.1\%) patients with multiple lesions while group 2 shows all $5(100 \%)$ patients to have multiple lesions (P value $=0.3$ ). All these data are shown in Table 1.

Multivariate regression analysis for different pathological variables (MRI positive findings, abnormal IMT, presence of carotid plaques and existence of I CAD) proved that abnormal MRI finding is the only independent predictor for MetS $(\mathrm{P}$ value $=0.002)$.

\section{Discussion}

The purpose of this study was to investigate and assess the atherosclerotic burden of MetS on extra and intracranial vasculature. This was achieved by determining whether MetS constitutes a significant difference in the risk of developing Carotid atherosclerosis and SBI or not and by detecting the proportion and pattern of asymptomatic Carotid atherosclerosis and SBI in MetS.

In the present study, extracranial low grade carotid atherosclerosis was significantly prevalent in participants with MetS compared to control subjects with single or more risk factors. However, the syndrome had no significant impact on isolated elevation of common carotid artery (CCA) mean intima media thickness (IMT). The

Table 1. Sonographic and MRI findings for the subjects enrolled in the study.

\begin{tabular}{|c|c|c|}
\hline & Group $1(\mathrm{~N}=50)$ & Group $2(\mathrm{~N}=30)$ \\
\hline Extracranial plaque with $<50 \%$ stenosis & $19(38 \%)$ & $4(13.3 \%)$ \\
\hline Unilateral plaque & $12(63.2 \%)$ & $4(100 \%)$ \\
\hline Bilateral plaques & $7(36.8 \%)$ & zero \\
\hline mean IMT & $0.09 \mathrm{~cm}$ & $0.08 \mathrm{~cm}$ \\
\hline Intracranial $<50 \%$ stenosis & $7(87.5 \%)$ & $3(100 \%)$ \\
\hline Intracranial $>50 \%$ stenosis & $1(12.5 \%)$ & zero \\
\hline Single vessel disease & $4(50 \%)$ & $2(66.7 \%)$ \\
\hline Multiple vessel disease & $4(50 \%)$ & $1(33.3 \%)$ \\
\hline SBI MRI brain & $18(75 \%)$ & $5(23.8 \%)$ \\
\hline Normal MRI brain & $6(25 \%)$ & $16(76.2 \%)$ \\
\hline Single lesion in MRI brain & $7(38.9 \%)$ & zero \\
\hline Multiple lesion in MRI brain & $11(61.1 \%)$ & $5(100 \%)$ \\
\hline
\end{tabular}


latter finding was somewhat unexpected and could be attributed to the lack of difference in the smoking status between both groups.

Several studies have examined the effect of MetS on asymptomatic extracranial atherosclerosis. Many of them concluded that MetS has a significant effect on both IMT elevation and the presence of atherosclerotic plaques. These findings may be accounted for by the difference in the population sample, range of age, different ethnicity, definition of MetS applied, sites of measuring and calculating mean Carotid IMT and adopting lower cutoff values for abnormal Carotid IMT [7] [9]-[12].

Regarding the intracranial vasculature, the current study demonstrates that MetS had no significant effect on the presence of intracranial atherosclerotic disease (ICAD), the degree of intracranial stenosis or the number of diseased intracranial vessels. López-Cancio et al. [13] assessed the risk factor profile between extracranial atherosclerotic disease (ECAD) and ICAD (including MetS). They concluded that MetS is an independent predictor of both ECAD and ICAD, this association was evidently higher with ICAD. The difference between their results and those of this study could be attributed mainly to their population sample (933 subjects) and different ethnicity (Caucasians) since they adopted the joint interim statement definition for MetS diagnostic criteria. To the best of our knowledge, there are limited data about the influence of MetS and intracranial atherosclerosis.

On the other hand, there was a significant association between Mets and SBIs detected by MRI studies.

This association between MetS and SBI has been extensively investigated by several authors. The current study results are in concordance with many of these studies [6] [14]-[17].

Cavalieri et al. [4] found no significant association between MetS and abnormal MRI findings (SBIs or WMLs), which was unexpected even to the authors. They attributed these results to the probability that they might have recruited healthier population than other studies that are in line with the present study such as [14] and [18].

The present study has several limitations. The population sample is small (80 subjects), the age range of subjects involved is not very wide (40 - 60 years old) and they belong to a single ethnic group (Egyptian). The Carotid IMT was measured only in one arterial segment which is the CCA (one, two and three $\mathrm{cm}$. proximal to the bifurcation bulb). An important limitation of this study is that we could not determine which of the five risk factors can independently predict extracranial atherosclerosis and SBI. A linear correlation between different vascular risk factors and the investigated variables (Carotid IMT, extracranial and intracranial atherosclerosis and SBI) could not be performed due to the fact that, apart from abdominal obesity, the other risk factors (DM, HTN and dyslipidemia) were diagnosed by either investigations or history if already diagnosed i.e. actual values weren't available for all subjects.

A point of strength in the present study is that we used the most recent definition of the MetS (consensus definition) which has set its cutoff values for different risk factors at lower levels than the WHO definition allowing more sensitive detection and diagnosis of risk factors involved. In addition, this definition comprises ethnic and gender specific cutoff values for detection of abdominal obesity, although currently there are no exact cutoff values available for diagnosing abdominal obesity among Middle Eastern/Mediterranean ethic groups (including Egyptians). So, according to the IDF MetS diagnostic criteria we had to use the European cutoff values which may not be the best applicable parameters for this population.

\section{Conclusion}

This study demonstrates that MetS does indeed have a more significant impact on causing atherosclerosis of extracranial large (in the form of atherosclerotic plaques) and small (in the form of SBI and WMLs) cerebral blood vessels than do individual risk factors separately. Thus, we can recommend that patients suffering from MetS need more aggressive medical therapy for prophylaxis against the occurrence of large artery atherosclerosis and consequently future overt cerebrovascular ischemic events like stroke and transient ischemic attacks (TIAs) or more subtle events such as mild cognitive impairment.

\section{References}

[1] Kassi, E., Pervanidou, P., Kaltsas, G., et al. (2011) Metabolic Syndrome: Definitions and Controversies. BMC Medicine, 9, 1-13. http://dx.doi.org/10.1186/1741-7015-9-48

[2] Alberti, K.G., Eckel, R.H., Grundy, S.M., et al. (2009) Harmonizing the Metabolic Syndrome: A Joint Interim Statement of the International Diabetes Federation Task Force on Epidemiology and Prevention; National Heart, Lung, and 
Blood Institute; American Heart Association; World Heart Federation; International Atherosclerosis Society and International Association for the Study of Obesity. Circulation, 120, 1640-1645. http://dx.doi.org/10.1161/CIRCULATIONAHA.109.192644

[3] Alberti, K.G., Zimmet, P. and Shaw, J. (2005) The Metabolic Syndrome-A New Worldwide Definition. The Lancet, 366, 1059-1062. http://dx.doi.org/10.1016/S0140-6736(05)67402-8

[4] Cavalieri, M., Ropele, S., Petrovic, K., et al. (2010) Metabolic Syndrome, Brain Magnetic Resonance Imaging, and Cognition. Diabetes Care, 33, 2489-2495. http://dx.doi.org/10.2337/dc10-0851

[5] Richart, T., Thijs, L., Nawrot, T., et al. (2011) The Metabolic Syndrome and Carotid Intima-Media Thickness in Relation to the Parathyroid Hormone to 25-OH-D3 Ratio in a General Population. American Journal of Hypertension, 24, 102-109. http://dx.doi.org/10.1038/ajh.2010.124

[6] Portet, F., Brickman, A.M., Stern, Y., et al. (2012) Metabolic Syndrome and Localization of White Matter Hyperintensities in the Elderly. Alzheimer's Dementia, 8, S88-S95. http://dx.doi.org/10.1016/j.jalz.2011.11.007

[7] Novo, S., Peritore, A., Trovato, R.L., et al. (2013) Preclinical Atherosclerosis and Metabolic Syndrome Increase Cardio- and Cerebrovascular Events Rate: A 20-Year Follow up. Cardiovascular Diabetology, 12, 155. http://dx.doi.org/10.1186/1475-2840-12-155

[8] Liberopoulos, E.N. and Elisaf, M.S. (2005) Diagnosis of the Metabolic Syndrome: Which Definition Should We Use ? Hellenic Journal of Cardiology, 46, 258-262.

[9] Sipilä, K., Moilanen, L., Nieminen, T., et al. (2009) Metabolic Syndrome and Carotid Intima Media Thickness in the Health 2000 Survey. Atherosclerosis, 204, 276-281. http://dx.doi.org/10.1016/j.atherosclerosis.2008.08.029

[10] Al-Nimer, M.S.M. and Hussein, I.I. (2010) Increased Mean Carotid Intima Media Thickness in Type 2 Diabetes Mellitus Patients with Non Blood Pressure Compartment Metabolic Syndrome: A Preliminary Report. International Journal of Diabetes in Developing Countries, 29, 19-22. http://dx.doi.org/10.4103/0973-3930.50710

[11] Tabatabaei-Malazy, O., Fakhrzadeh, H., Sharifi, F., et al. (2012) Gender Differences in Association between Metabolic Syndrome and Carotid Intima Media Thickness. Journal of Diabetes \& Metabolic Disorders, 11, 13. http://dx.doi.org/10.1186/2251-6581-11-13

[12] Won, K., Chang, H., Kim, H., et al. (2013) Differential Impact of Metabolic Syndrome on Subclinical Atherosclerosis According to the Presence of Diabetes. Cardiovascular Diabetology, 12, 41. http://dx.doi.org/10.1186/1475-2840-12-41

[13] López-Cancio, E., Galán, A., Dorado, L., et al. (2012) Biological Signatures of Asymptomatic Extra- and Intracranial Atherosclerosis: The Barcelona-AsIA (Asymptomatic Intracranial Atherosclerosis) Study. Stroke, 43, 2712-2719. http://dx.doi.org/10.1161/STROKEAHA.112.661702

[14] Kwon, H.M., Kim, B.J., Lee, S., et al. (2006) Metabolic Syndrome as an Independent Risk Factor of Silent Brain Infarction in Healthy People. Stroke, 37, 466-470. http://dx.doi.org/10.1161/01.STR.0000199081.17935.81

[15] Bokura, H., Yamaguchi, S., Iijima, K., et al. (2008) Metabolic Syndrome Is Associated with Silent Ischemic Brain Lesions. Stroke, 39, 1607-1609. http://dx.doi.org/10.1161/STROKEAHA.107.508630

[16] Park, K., Yasuda, N., Toyonaga, S., et al. (2008) Significant Associations of Metabolic Syndrome and Its Components with Silent Lacunar Infarction in Middle Aged Subjects. Journal of Neurology, Neurosurgery and Psychiatry, 79, 719-721. http://dx.doi.org/10.1136/jnnp.2007.134809

[17] Kwon, H.M., Kim, B.J., Park, J.H., et al. (2009) Significant Association of Metabolic Syndrome with Silent Brain Infarction in Elderly People. Journal of Neurology, 256, 1825-1831. http://dx.doi.org/10.1007/s00415-009-5201-8

[18] Park, K., Yasuda, N., Toyonaga, S., et al. (2007) Significant Association between Leukoaraiosis and Metabolic Syndrome in Healthy Subjects. Neurology, 69, 974-978. http://dx.doi.org/10.1212/01.wnl.0000266562.54684.bf 